\title{
Genome-enabled discovery of evolutionary divergence in brains and behavior
}

Chinar Patil ( $\nabla$ cpatil6@gatech.edu )

Georgia Institute of Technology

Jonathan Sylvester

Georgia State University

Kawther Abdilleh

Georgia Institute of Technology

Michael W. Norsworthy

Catalog Technologies

Karen Pottin

Institut de Biologie Paris-Seine

Milan Malinsky

University of Basel

Ryan F. Bloomquist

Augusta University

Zachary V. Johnson

Georgia Institute of Technology

Patrick T. McGrath

Georgia Institute of Technology

Jeffrey T. Streelman

Georgia Institute of Technology

\section{Research Article}

Keywords: Malawi Cichlids, genome sequencing, evolutionary reverse genetics, brain development, behavior

Posted Date: March 12th, 2021

DOl: https://doi.org/10.21203/rs.3.rs-275291/v1

License: (c) (i) This work is licensed under a Creative Commons Attribution 4.0 International License. Read Full License 
1 Genome-enabled discovery of evolutionary divergence in brains and

2 behavior

3 Chinar Patil ${ }^{4}$, Jonathan B. Sylvester ${ }^{1,2}$, Kawther Abdilleh ${ }^{1}$, Michael W. Norsworthy $y^{3,4}$,

4 Karen Pottin ${ }^{5}$, Milan Malinsky,, , Ryan F. Bloomquist' ${ }^{1,8}$, Zachary V. Johnson ${ }^{1}$, Patrick T.

5 McGrath', Jeffrey T. Streelman ${ }^{1}$

6

7 Affiliations:

$8{ }^{1}$ School of Biological Sciences and Petit Institute of Bioengineering and Bioscience, 9 Georgia Institute of Technology, Atlanta, GA, USA.

10 'Department of Biology, Georgia State University, Atlanta, GA, USA

$11{ }^{3}$ Catalog Technologies Inc., Boston, MA, USA.

12 4Freedom of Form Foundation, Inc., Cambridge, MA, USA.

13 5Laboratoire de Biologie du Dévelopement (IBPS-LBD, UMR7622), Sorbonne Université, 14 CNRS, Institut de Biologie Paris Seine, Paris, France.

15 'Zoological Institute, Department of Environmental Sciences, University of Basel, Basel, 16 Switzerland.

17 7Wellcome Trust Sanger Institute, Cambridge, United Kingdom.

$18{ }^{8}$ Augusta University, Department of Oral Biology and Diagnostic Sciences, Department

Corresponding Author:

Chinar Patil

Email: cpatil6@gatech.edu

Keywords: Malawi Cichlids, genome sequencing, evolutionary reverse genetics, brain development, behavior 


\section{Abstract}

30 Lake Malawi cichlid fishes exhibit extensive divergence in form and function built from a

31 relatively small number of genetic changes. We compared the genomes of rock- and

32 sand-dwelling species and asked which genetic variants differed among the groups. We

33 found that $96 \%$ of differentiated variants reside in non-coding sequence but these non-

34 coding diverged variants are evolutionarily conserved. Genome regions near

35 differentiated variants are enriched for craniofacial, neural and behavioral categories.

36 Following leads from genome sequence, we used rock- vs. sand- species and their

37 hybrids to (i) delineate the push-pull roles of BMP signaling and irx $1 b$ in the specification

38 of forebrain territories during gastrulation and (ii) reveal striking context-dependent brain

39 gene expression during adult social behavior. Our results demonstrate how divergent

40 genome sequences can predict differences in key evolutionary traits. We highlight the

41 promise of evolutionary reverse genetics - the inference of divergence in phenotype from

42 genome sequencing in natural populations. 


\section{Introduction}

44 Our understanding of how the genome encodes natural variation in form and function is 45 still limited. This is the case for almost any trait, from height to behavior to complex disease ${ }^{1}$. The reasons for this are manifold, but they include an underappreciated role of non-coding genetic variants linked to differences in traits. This is apparent in our assumptions and in syntheses of data. For instance, only 25 years ago, experts thought that the human genome might contain 70,000 to over 100,000 genes to account for our complexity ${ }^{2}$. More recently, it has been estimated that upwards of $93 \%$ of human disease related variants - traits for which we have the most data from genome wide association studies (GWAS) - reside in noncoding DNA sequence ${ }^{3}$. Many of these noncoding variants are regulatory, that is, they affect the expression of genes ${ }^{4}$. Therefore, despite a refined understanding of how single genes work in controlled cellular environments, it remains unclear how the genome is activated to produce natural phenotypes, and this may be particularly vexing for context-dependent processes like development or 57 behavior.

Over the past two decades, systems have been developed to identify the genetic basis of traits from nature ${ }^{5}$. Amongst vertebrate animals, these traits include body armor ${ }^{6}$, color ${ }^{7}$, head and jaw shape ${ }^{8-10}$, parental care ${ }^{11,12}$, song ${ }^{13}$ and coordinated movement 14. The take home message from this work has been that a small number of genes from 63 recognizable pathways explain a considerable proportion of phenotypic variance. Yet, 64 these studies may be biased in interpretation and limited in inference space. The focus is 65 typically on one or two species and one trait at a time, often using hybrid pedigrees 66 founded by a small number of individuals, and candidate gene or QTL approaches. Here, 67 we explored a different strategy in a system of many species with many divergent traits. 68 We sought to determine the genetic differences between closely related groups of species 69 and then to focus experiments on leads from genome divergence. In essence, we've asked the genome which traits to follow.

72 The Malawi cichlid system is an apposite one for our research aims. The assemblage 73 comprises hundreds of closely related species that have diversified in the last 500,000 to 
74 one million years ${ }^{15}$, such that the genomes of individuals across species boundaries 75 remain highly similar ${ }^{16}$. An appreciable fraction of genetic polymorphism identified in 76 Malawi species is shared with cichlid lineages from throughout East Africa -- suggesting 77 that ancient genetic variation fuels diversification of the Malawi flock ${ }^{17}$. Set against this 78 background of genome similarity, Malawi cichlids exhibit staggering diversity in 79 phenotypes including pigmentation ${ }^{18}$, sex determination ${ }^{19,20}$, craniofacial and brain 80 patterning 8,21,22 and social behavior ${ }^{23-25}$. Previous work has focused on the genomic and 81 early developmental underpinnings of this diversity, in rock- vs. sand-dwelling species $8216,21,22,26$.

84 Rock- vs. sand- species form ecologically distinct groups similar to other ecotypes in well85 known adaptive radiations (marine vs. freshwater sticklebacks; tree vs. ground finches and anoles) ${ }^{27}$. The main difference in this case is that each of the rock-and sand-groups

87 contains more than 200 species. Recent divergence, rapid speciation and meta88 population dynamics synergistically lead to the broad sharing of polymorphism across the rock-sand speciation continuum ${ }^{17,28}$. Malawi rock-dwellers tend to be strongly territorial 90 and aggressive; they breed and feed at high density in complex rock-reef habitats. Most 91 eat algae from the substratum with strongly reinforced jaws packed with teeth. Adult rock92 dweller brains exhibit enlarged anterior components, telencephala and olfactory bulbs.

93 Sand-dwellers are less site-specific and less aggressive. They often breed on communal 94 leks where males build sand 'bowers' to attract females ${ }^{29}$. Many capture small prey using 95 acute vision and fast-moving gracile jaws; their brains and sensory apparatus are 96 elaborated for more posterior structures optic tecta, thalamus and eyes (Supplementary 97 Figure 1). We aimed to understand evolutionary divergence between rock- and sand98 dwelling lineages by identifying the number, type and spectrum of genetic variants that 99 separate these groups.

101 To target this primary axis of evolutionary divergence in the Lake Malawi species 102 assemblage ${ }^{27}$, we compared whole genomes of one male individual each from 8 rock103 dwelling and from 14 sand-dwelling species (Supplementary Table 1), to an average of $10425 \mathrm{X}$ coverage per individual. Species were chosen to represent the diversity present 
105 within each of the rock- and sand- groups (Figure 1A), in terms of body size, color pattern, 106 ecology and phylogenetically defined lineages within the sand-species group ${ }^{28}$. 
108 The genomic signature of rock-sand divergence

109 We compared the genomes of 8 rock dwellers and 14 sand dwellers to uncover the 110 genomic signature of rock- versus sand- evolutionary diversification. We aligned 111 sequence data to a reference genome of nearly 1 gigabase 30 and identified 112 approximately 22 million Single Nucleotide Polymorphisms (SNPs) and 200,000 113 Insertion-Deletions (InDels). We calculated Fst per variant, and averaged across 10kb 114 windows, to quantify divergence between rock and sand species. We found that $0.06 \%$ 115 of SNPs and $0.44 \%$ of InDels are alternately fixed between rock- and sand- groups. When 116 these divergent variants and genome regions (2.5\% FDR) are mapped to linkage groups 117 (chromosomes), it is apparent that the signature of rock- vs. sand- divergence is 118 distributed relatively evenly across the chromosomes (Figure 1B). Among fixed variants, $1193.5 \%$ were found in coding regions and 96.5\% were predicted to be non-coding; $\sim 17 \%$ in 120 intergenic regions, $38 \%$ in introns, 38\% in flanking regions (within $25 \mathrm{~kb}$ up- or 121 downstream of a gene), and 3\% in annotated UTRs. Rock vs. sand fixed coding variants 122 were more likely to be missense/loss-of-function (72.6\%) than silent (27.3\%).

124 We next generated whole-genome alignments of five published cichlid reference 125 genomes from across East Africa ${ }^{31}$ and estimated an evolutionary conservation score for 126 each nucleotide position. Akin to phylogenetic footprinting, this approach allows inference 127 of function for regions that are slower to change than others due to the long-term effect 128 of purifying selection. For both coding and non-coding portions of the genome, we found 129 that rock-sand divergence correlates positively with evolutionary conservation scores

130 (Figure 1C), suggesting that differentiated rock-sand variants, including many non-coding 131 variants, are enriched for function.

133 A total of 4,484 genes lie within $25 \mathrm{~kb}$ of either an alternately fixed variant or a highly 134 divergent $10 \mathrm{~kb}$ window (2.5\%FDR). Pathway enrichment analysis 32 of human 135 homologs/analogs for these genes reveals categories spanning early embryonic 136 development, craniofacial morphogenesis, brain development, synaptic transmission and 
137 neuronal function (Supplementary Table 2). In particular, rock-sand divergent genes are 138 enriched for GO Biological Process terms 'telencephalon development' ( $p<1.7 e-18)$, 139 'adult behavior' $(p<2 e-14)$, 'synaptic plasticity' $(p<1.4 e-12)$, 'odontogenesis' $(p<3.7 e-$ 140 11), 'response to BMP' ( $p<3.2 e-09)$, 'gastrulation' $(p<5.6 e-06)$, 'face morphogenesis' 141 ( $p<8.9 e-08)$, 'neural crest cell differentiation' $(p<4.3 e-13)$, and 'eye development' $(p<$ 142 1.3e-15). Over-represented gene families included nuclear hormone receptors $(p<3.0 \mathrm{e}-$ 143 08), HOXL subclass homeoboxes ( $p<1.4 \mathrm{e}-07)$, TALE class homeoboxes $(p<4.2 \mathrm{e}-04)$ 144 and Forkhead boxes ( $p<9.33-04$; for novel expression domains in cichlid foxp2 see 145 Supplementary Figure 2). We observed enrichment for the mouse phenotypes 'abnormal 146 cognition' ( $p<3.3 e-15$ ), 'abnormal learning and memory' ( $p<2.6 e-15)$, 'abnormal 147 craniofacial morphology' $(p<4.9 \mathrm{e}-11)$ and 'abnormal social/conspecific interaction' $(p<$ 148 7.1e-14). We used the list of differentiated genes to query an Allen Brain Atlas dataset 149 that reports gene expression in hundreds of brain regions ${ }^{33}$. Rock- sand- divergent genes 150 were enriched for the basomedial nucleus of the amygdala, a sub-region of the 151 telencephalon ( $p_{\text {adj }}=0.001$ ) that regulates fear, anxiety, and physiological responses to 152 territorial intruders in rodents ${ }^{34,35}$, and has been linked to Social Anxiety Disorder in 153 humans ${ }^{36}$. Finally, we queried databases of genes involved in human disease. Genes 154 near divergent variants are significantly enriched for factors implicated in neurological 155 disease like Autism Spectrum Disorder (SFARI ${ }^{37}$, Fisher's exact test $p$ value $<2 e-16$ ) 156 and disorders related to the neural crest ${ }^{38}$, (Fisher's exact test $p$ value $<2 e-16$ ).

158 Given the prevalence of evolutionarily conserved, non-coding, divergent rock-sand 159 variants and genome-wide enrichment for craniofacial and neural crest biology, we 160 examined overlap with published datasets of mammalian neural crest and craniofacial 161 enhancers ${ }^{39,40}$. These data allow us to identify craniofacial and cranial neural crest cell 162 (CNCC) enhancers conserved between mammals and cichlids and fixed variants 163 between rock and sand species within these conserved regulatory elements. A total of 164275 craniofacial enhancer elements and 234 human CNCC enhancers are evolutionarily 165 conserved between mammals and cichlids. We found divergent rock-sand mutations 166 within the enhancer elements of key genes integral to CNCC specification and migration 167 (Supplementary Table 2). Notably, from both datasets, fixed rock-sand variants were 
168 found within the enhancer region of the gene $n r 2 f 2$, a nuclear receptor and master neural 169 crest regulator ${ }^{41}$. Rock-sand divergent variants were similarly located within craniofacial 170 enhancers of three genes (yap1, fat4, rere) that function in the Hippo signaling pathway, 171 as well as within enhancers of irx3 and axin2. These data linking rock-sand fixed 172 SNP/InDels to evolutionarily conserved, experimentally verified enhancers further 173 underscore the importance of non-coding variation in the craniofacial evolution of rock174 and sand- lineages ${ }^{42}$.

175

176 Genome-wide divergence between rock vs. sand Malawi cichlids involves a relatively 177 small percentage of genetic variants. Divergent variants are (a) predominantly non178 coding, (b) in long-term evolutionarily conserved loci (c) enriched for genes and pathways 179 involved in embryonic development, brain development, brain function and behavior, and 180 craniofacial morphogenesis. Given these strong patterns of enrichment, we used the 181 experimental power of the Malawi cichlid system to interrogate features of early 182 development and adult behavior that differ between rock- and sand- groups.

\section{A gastrula-stage map of forebrain diversification}

185 Rock- vs. sand-dwelling Malawi cichlids exhibit divergence in or near genes enriched for 186 BMP signalling, gastrulation, eye and telencephalon development, as well as the TALE 187 (Irx) gene family. To explore the developmental consequences of this differentiation, we 188 investigated early forebrain specification in rock- and sand- embryos, building upon our 189 previous studies and interest in irx $1 b$ and early brain development ${ }^{21,22}$. During 190 development, the complexity of the vertebrate brain is first laid out in the neural plate, a

191 single-cell thick sheet of cells that forms between non-neural ectoderm and the germ ring 192 at gastrulation. Irx genes act as transcriptional repressors of BMP signal in gastrulation, 193 and function to specify the neural plate ${ }^{43}$. BMPs, in turn, are protective of the anterior194 most region of the neural plate, which will ultimately give rise to the telencephalon, and 195 suppress the eye field ${ }^{44}$. Given alternatively fixed variants in the irx $1 b$ gene, expected 196 interactions between Irx and BMP signaling in the early embryo and known telencephalon 
197 vs. eye size differences between rock- vs. sand- species 21,22, we examined and 198 quantified the early activity of irx $1 b$ and BMP in rock- vs. sand- embryos.

200 We used a custom device to orient and image cichlid embryos in toto at gastrula and 201 neurula stages ${ }^{45}$. In early gastrula (EG), irx1b (red) and BMP signal (green, PSMAD) 202 delineate complementary dorsal and ventral domains of the embryo (Figure 2A). By mid203 gastrula (MG), irx1b shows two expression domains, one in the posterior portion of the 204 developing neural plate (np) and the second co-expressed with PSMAD activity around 205 its anterior border (white arrowheads). By late gastrula (LG), the domains of $i r \times 1 b$ 206 expression and PSMAD activity sharpen around the leading edge of the neural plate but 207 remain overlapping around the periphery. Notably, irx1b expression is expanded in the 208 anterior domain of sand-dwellers (S) compared to rock-dwellers at EG and MG, and then 209 defines the boundary of the neural plate earlier in sand-dwellers (S) in LG (arrowheads). 210 As a consequence, BMP signal should have a longer-lasting influence on the neural plate 211 in rock-dwelling species. Based upon manipulative experiments in zebrafish ${ }^{44}$, this is 212 predicted to result in a relatively larger presumptive telencephalon and smaller eye field.

214 We developed a panel of rock- $x$ sand- hybrid crosses to formally evaluate the role of $215 i r \times 1 b$ in forebrain diversification. First, we used quantitative RT-PCR to measure allele216 specific expression (ASE) in heterozygous rock- $x$ sand- $F_{2}$ hybrids, across the stages of 217 gastrulation. We observed that the sand-irx1b allele was expressed at significantly higher 218 levels (average of 2.5-fold; $p=4.5 e-13$; Student's t-test) and that this difference was 219 largely confined to MG (Figure 2B). Next, we used hybrid embryos to chart the 220 development of the telencephalon and the eye field. Rock- $x$ sand- $F_{2}$ hybrids, indexed 221 for irx $1 b$ genotype, were raised to neurula and somitogenesis stages and we examined 222 the expression of shh (which induces foxg1 and the ventral forebrain), foxg1 (a marker of 223 the telencephalon), and $r \times 3$ (a marker of the eye field), by in situ hybridization. $F_{2}$ 224 individuals homozygous for rock- irx $1 b$ alleles exhibited a larger and more rostral domain 225 of shh expression, an earlier and larger domain of foxg1 and a smaller rx3 domain (Figure 226 2C, D). These differences between rock- vs. sand- irx1b genotypes match expression 227 divergence observed amongst rock- vs. sand- species ${ }^{21,22}$. Finally, when we compared 
228 the relative size of the telencephalon among irx $1 b$ genotypes, individuals homozygous

229 for rock- alleles exhibited larger telencephala (Supplementary Figure 3). We conclude

230 that genetic variants in and around the $i r \times 1 b$ gene contribute to divergent specification of

231 the Malawi cichlid forebrain, likely via spatial, temporal and quantitative variation in the

232 expression of irx $1 b$ itself.

234 Our genome sequencing revealed a near-fixed InDel in the 3' UTR of the Malawi cichlid 235 irx1b gene (Supplementary Figure 4). Rock- species possess an 85bp insertion, 236 compared to cichlid species from outside of the Malawi lineage. Sand-dwellers largely 237 lack the insertion and exhibit a $6 \mathrm{bp}$ deletion compared to outgroups. The insertion shows 238 strong genetic similarity to a fragment from the Rex 1 family of non-LTR retrotransposons $239{ }^{46}$. Given the likelihood that Astatotilapia calliptera populations surrounding Lake Malawi 240 may have seeded the Malawi evolutionary radiation and contributed to rock- and sand241 dwelling lineages ${ }^{17,28}$, we explored the presence/absence of this InDel in Astatotilapia 242 samples. We found that most Astatotilapia individuals and populations had the rock- ir $1 \mathrm{~b}$ 243 allele (the insertion), but that an individual from Chizumulu Island was fixed for the sand244 allele and two individuals sampled from Itupi were heterozygous. Because the 85bp 245 insertion in rock- species is a partial Rex1 fragment, and sand- species carry a 6bp 246 deletion compared to outgroups, we speculate that the current rock- and sand- divergent 247 alleles were generated by at least two imperfect excision events of an element that 248 invaded the genome of the Malawi + Astatotilapia ancestor. Rex1/Babar retrotransposons 249 have been active in African cichlid genomes, and are known to influence gene expression 250 when inserted in 5' and 3' UTRs ${ }^{31}$. Future experiments will determine whether this Rex1 251 insertion causes the differences in $i r x 1 b$ gene expression and forebrain specification we 252 observed.

254 Genomics of divergent social challenge and opportunity

255 Rock- and sand-dwelling Malawi cichlids live in strikingly different social and physical 256 environments. Rock-dwelling males tend to be more aggressive than sand-dwellers ${ }^{23}$ 257 and defend territories year-round as sites for feeding and breeding. By contrast, sand- 
258 dwellers are more exploratory than rock-dwellers ${ }^{25}$ and only breeding males tend to be 259 territorial, often building sand bowers to attract females and mitigate male-male 260 aggression. Given these observations and genome-wide enrichment for categories 261 related to adult behavior and social interaction, we designed an experimental paradigm 262 to investigate brain gene expression profiles associated with divergent rock- vs. sand263 social behaviors.

We evaluated social challenge and opportunity amongst males using a large tank with a 'rock' habitat at one end and 'sand' at the other, separated by glass bottom (Figure 3A). When parental rock- species are placed in this tank paradigm, males court females on the rock side of the tank. Males of sand- species court females over sand and construct species-appropriate bowers. When single hybrid rock- $x$ sand- $F_{1}$ males are placed in this arena with hybrid $F_{1}$ females, males invariably court females over the 'rock' habitat. However, when two rock- $x$ sand- hybrid $F_{1}$ males (brothers) were allowed to compete for gravid hybrid $F_{1}$ females in this tank paradigm, we observed something different. One male, typically the larger, courted females over the rock habitat, and the other

274 simultaneously constructed bowers to court females over the sand. We found no 275 difference in gonadal-somatic index (GSI), an established biological metric of 276 reproductive status and maturity, between $F_{1}$ males behaving as 'socially rock' vs. 277 'socially sand.' (Supplementary Figure 5). Our observation of divergent behavior between $278 \quad F_{1}$ brothers in the same tank suggests an interaction between the genome and the social 279 environment.

We used RNA-seq to investigate gene expression profiles associated with behavior of 282 rock- $x$ sand- $F_{1}$ hybrid males that were actively courting females over rock vs. sand. 283 Whole brains of $F_{1}$ males tested singly ( $n=2$ lone) as well as $F_{1}$ brothers assayed in dyads 284 ( $\mathrm{n}=4$ dyads) were collected during courtship, and interrogated by RNA-seq. Strikingly, gene expression profiles clustered not by fraternal relatedness, but rather by behavioral 286 context (Figure 3B). Males from dyads that courted females over rocks had expression 287 profiles similar to single males (who also courted over rocks) but distinct from their brothers that built bowers and courted females over sand in the same tank. Genes were 
289 considered significantly differentially expressed between 'social rock' and 'social sand' 290 brains if they exhibited both a fold change $\geq 2$ and crossed the threshold of $p_{a d j}<0.05$. 291 Based on this criterion, we found 832 genes differentially expressed between rock- vs. 292 sand-behaving males (Figure 3B, Supplementary Table 3). Among differentially 293 expressed genes, we observed significant functional enrichment for GO Biological 294 Process categories 'synaptic signaling' ( $p<2.3 e-21)$, 'synaptic plasticity' $(p<3.6 e-09)$, 295 'visual behavior' $(p<2.09 \mathrm{e}-06)$; mouse phenotypes 'abnormal 296 learning/memory/conditioning' ( $p<5.9 e-07)$, 'abnormal telencephalon morphology' $(p<$ 297 3.95e-07), 'abnormal spatial learning' ( $p<9.9 e-07)$ and pathways 'axon guidance' ( $p<$ 298 3.3e-05), 'oxytocin signaling' ( $p<5.2 e-05)$ and 'estrogen signaling' $(p<1.8 e-04)$ 299 (Supplementary Table 3). Matches against the Allen Brain Atlas database of gene 300 expression yielded enrichment for exclusively sub-regions of the telencephalon: CA3, 301 hippocampus ( $\left.p_{a d j}=4.6 e-6\right), C A 2$, hippocampus ( $\left.p_{a d j}=7.3 e-5\right), C A 4$, hippocampus ( $p_{a d j}$ $302=0.001)$, claustrum $\left(p_{a d j}=0.001\right)$, subiculum $\left(p_{a d j}=0.003\right)$, dentate gyrus $\left(p_{a d j}=0.03\right)$ 303 and the basomedial nucleus of the amygdala ( $p_{a d j}=0.03$ ). The hippocampus encodes 304 episodic memory and spatial representations of the environment ${ }^{47}$, and more recently its 305 subregions have been shown to play critical roles in anxiety, social interaction, and social 306 memory formation ${ }^{48-50}$. Roughly $38 \%$ of differentially expressed genes also contained 307 genetically differentiated SNP/InDels between rock- and sand- species ( $p$-value $<2 e-6$, 308 Fisher's exact test), implying considerable cis-acting genetic variation. Enrichment of 309 numerous categories related to brain function and synaptic plasticity showed greater 310 overlap than expected (Figure 3C). These context-dependent differences suggest 311 concerted changes in brain gene expression as males experienced and responded to 312 different social challenges and opportunities ${ }^{24,51}$. 


\section{Discussion}

314 A fundamental problem in evolutionary biology is understanding the cellular,

315 developmental and genetic basis of how traits change. This is a challenge because we

316 lack sufficient information about how genes work in outbred genomes from nature and we

317 do not fully comprehend the causal role of noncoding variation in specifying form and

318 function. This problem is especially difficult for traits that are only observed in particular

319 contexts, like development and behaviour. To make progress, we and others have 320 focused on study systems exhibiting abundant phenotypic diversity built from a relatively

321 small number of genetic changes. Here we identify and characterize the genetic variants

322 that demarcate one of the deepest evolutionary splits amongst Lake Malawi cichlid 323 groups, that between rock- and sand-dwelling species thought to have diverged in the 324 last one million years. We found a small percentage (less than $0.1 \%$ ) of genetic variants 325 to be differentiated between rock- and sand-groups, and that the majority of differentiated 326 variants (>96\%) were noncoding. Differentiated non-coding variants were more likely to 327 be in an evolutionarily conserved locus as a function of genetic differentiation, suggesting 328 that divergent rock- vs. sand- noncoding changes are functional. To support this idea, we 329 identified alternately fixed rock- vs. sand- noncoding variants within experimentally 330 verified, vertebrate-conserved craniofacial and cranial neural crest cell enhancers. The 331 latter observation is similar in type to the discovery of human-specific deletions within 332 mammal-conserved regulatory sequence ${ }^{52}$.

334 Recently we surveyed genome-wide divergence between sand-dweller sub-groups that 335 construct pit vs. castle bowers, sand-made structures to attract females for mating ${ }^{24}$. 336 Mapping those variants to the same genome reference, we expected distinct patterns of 337 diversification because rock-sand and pit-castle divergence likely occurred at different 338 times, along different trait axes, under the control of different evolutionary forces 27. 339 Consistent with expectation, there is clear clustering of genome divergence on 340 chromosome 11 for the pit-castle comparison (Supplementary Figure 6), while all 341 chromosomes carry the signature of rock-sand diversification (Figure 1B). However, 342 contrary to our expectation, rock- vs. sand- and pit- vs. castle- radiations have diverged 343 in similar gene sets. Out of 3070 genes identified near 10kb high Fst regions in the rock- 
344 vs. sand- comparison, 483 overlap with 1090 genes identified near high Fst regions in 345 the pit- vs. castle- comparison ( $p$-value $<2 e-9$, Fisher's exact test, Supplementary Table 346 4). This result may imply that evolutionary diversification in Lake Malawi is limited, or 347 constrained, by chromosomal location.

349 Overall, genes in proximity to rock-sand divergent variants were enriched for functional 350 categories related to early forebrain and craniofacial development, neuronal function and 351 social behavior. This list of variants, coupled with consistent patterns of functional and 352 pathway enrichment, motivated follow up experiments focused on early brain 353 development and adult social behavior (Figure 4). It is apparent from our work here and 354 previously ${ }^{21,22}$, that Malawi cichlid brains and nervous systems begin to differ during gastrulation in pathways that can be predicted from divergent genome sequences. This 356 is interesting for at least two reasons. First, this observation runs counter to the late 357 equals large' textbook example ${ }^{53}$ of how brains evolve differences in relative proportions 358 of their parts ${ }^{22}$. Similarly, such early variation in development is not thought to be a driving 359 force in evolution, precisely because early changes can have global and ramifying effects. 360 Collectively, our findings provide a partial description of the conditions wherein variation 361 during the earliest stages of development can contribute to evolutionary diversification. In 362 each case we have examined, variation in gene expression is quantitative, heterochronic 363 and limited to a precise stage or time period.

365 Sydney Brenner recognized the relationship between the genetic specification of nervous 366 systems and the behavioral output of the brain ${ }^{54}$. However, because these events take 367 place so far apart in the lifespan of a vertebrate, they are rarely studied simultaneously. 368 Here, the genome connects the two phenomena: rock- vs. sand- divergent gene sets 369 indicating that both brain development and social behavior have been under divergent 370 selection during the evolutionary diversification of these groups. To evaluate social 371 behavior in rock- vs. sand-dwelling Malawi cichlids, we constructed a social context 372 arena. The presence of sand and simulated rocky caves was sufficient to elicit species373 appropriate male behavior when rock- or sand- males were tested with rock- or sand374 gravid females. When rock- $x$ sand- $F_{1}$ hybrid males were tested, one per tank, with $F_{1}$ 
375 hybrid females, males courted females in the rock quadrant of the tank. Notably, when 376 dyads of $F_{1}$ brothers were tested in this tank paradigm with gravid $F_{1}$ females, we 377 observed simultaneous 'social rock' and 'social sand' behavior. Brain gene expression 378 profiles from behaving males clustered by social context (social rock vs social sand), and 379 not by fraternal relationships. Differentially expressed genes were enriched for brain 380 regions and pathways implicated in social interaction and overlapped significantly with 381 rock- vs. sand- divergent genetic variants.

383 Social context is known to influence the brain. For instance, our clustering results are 384 similar to those of Whitfield and colleagues (Whitfield et al. 2003) who showed that brain 385 gene expression in honey bees was predictive of behavior. Likewise, changes in brain 386 morphology and gene expression predictably accompany the ascent to dominance in the 387 cichlid fish Astatotilapia burtoni ${ }^{55}$. Our data seem not to fit the model of dominant388 subordinate however. In our experiments, the gonado-somatic index (GSI) did not differ 389 between social rock vs. social sand brothers within dyads. Both males exhibited nuptial 390 coloration, courted females and in cases with multiple gravid females, both brothers 391 reproduced. Body size was associated with divergent social rock vs. social sand behavior 392 of $F_{1}$ males; the social rock brother was always larger (mean mass was $26.96 \mathrm{~g} \pm 3.4$ [SE] 393 compared to $19.45 \mathrm{~g} \pm 2.2)$.

395 Our experiments demonstrate that $F_{1}$ hybrid male brains can express both social rock396 and social sand- behavioral programs, and that social context determines which program 397 is executed. This observation is similar to, but also different than, pit-digging $x$ castle398 building $F_{1}$ male Malawi cichlids who carry out parental bower behaviors in a specific 399 sequence ${ }^{24}$. Notably, in both cases, the hybrid males exhibit one of the two different 400 parental behaviors at any one time - there is no intermediate behavior. In the pit- vs. 401 castle- case, we think that the bower structure itself and/or a threshold signal from females 402 might lock the hybrid male brain into a behavioral state. In the rock- vs sand- case here, 403 it appears that other social cues (i.e., the presence and size of a rival male) lock the hybrid 404 male brain into a behavioral state. These context-dependent behaviors, accompanied by 405 changes in brain gene expression, are compelling examples of interaction between the 
406 genome and the social environment. The cellular and genetic basis of these behaviors 407 and their plasticity deserves further attention. Our comparative genomic and brain gene 408 expression data, combined with enrichment testing and experimental approaches, 409 highlight that the Malawi cichlid telencephalon will be central to this future work. 


\section{Genome sequencing}

412 We extracted genomic DNA, from fin clips of 22 male individuals (Qiagen DNeasy, Cat 413 \#69504), from 8 rock dwelling and 14 sand dwelling Lake Malawi species (Supplementary 414 Table 2) representing broad diversity across the rock and sand lineages in Lake Malawi.

415 We made libraries using the Illumina Nextera Library prep kit and performed paired-end 416 sequencing on the Illumina Hi-Seq 2500 at Georgia Tech. The Metriaclima zebra 417 reference genome version $M Z_{\text {_U UMD2a }}{ }^{30}$ was used for genome alignment, variant 418 discovery and annotation using standard BWA ${ }^{56}$ and GATK practices ${ }^{57}$. The maximum 419 likelihood tree in Figure 1A was constructed using SNPhylo ${ }^{58}$, from variant data.

Genetically Divergent Regions

422 Vcftools ${ }^{59}$ was used to calculate FST (--weir-fst-pop) between the 8 rock and 14 sand 423 species. Variants with FST $=1$ were noted to be alternately fixed between rock and sand 424 lineages in our dataset. FST was also measured across 10kb windows (--fst-window-size). 425 Significance thresholds were marked using the fdrtool package in R. All variants were 426 annotated using Snpeff $4.3 \mathrm{i}{ }^{60}$. We tested the genes within $25 \mathrm{~kb}$ of significantly 427 differentiated variants for enrichment of functional categories. The cichlid gene names 428 were converted to human analogs using Treefam based mapping ${ }^{61}$ and functional 429 enrichment was determined using the TOPPFUN web-browser interface ${ }^{62}$.

431 PhastCons analysis

432 Pairwise alignments were generated using lastz $\mathrm{v} 1.02^{63}$, with the following parameters: 433 " $\mathrm{B}=2 \quad \mathrm{C}=0 \quad \mathrm{E}=150 \quad \mathrm{H}=0 \quad \mathrm{~K}=4500 \mathrm{~L}=3000 \quad \mathrm{M}=254 \quad \mathrm{O}=600 \quad \mathrm{Q}=$ human_chimp.v2.q $\mathrm{T}=2$ $434 \mathrm{Y}=15000$ ". This was followed by using USCS genome utilities 435 (https://genome.ucsc.edu/util.html,

436 https://hgdownload.soe.ucsc.edu/admin/exe/linux.x86_64/FOOTER) axtChain tool with 437 minScore $=5000$. Additional tools with default parameters were then used following the 
439 (http://genomewiki.ucsc.edu/index.php/Whole_genome_alignment_howto) in order to 440 obtain a contiguous pairwise alignment. Multiple alignments were generated from 441 pairwise alignments with the multiz v11.2 ${ }^{64}$ program, using default parameters and the 442 following pre-determined phylogenetic tree: ((( $M$. zebra, P. nyererei), A. burtoni), $N$. 443 brichardi), O. niloticus) in agreement with Brawand et al. ${ }^{31}$. Sequence conservation 444 scores were then obtained using PhastCons ${ }^{65}$ with a phylogenetic model estimated by 445 the phyloFit ${ }^{66}$ program, both from the PHAST software package (v.1.3). The model fitting 446 was done using default parameters. PhastCons was run in two iterations, first to obtain 447 the free parameters of the model (--estimate-trees and --no-post-probs) and then using 448 the output from this we ran PhastCons again to attain the conservation scores with -449 target-coverage 0.3 --expected-length 100.

\section{Vertebrate-conserved enhancer elements}

452 A comparative genomic approach was used to identify putative craniofacial and neural 453 crest CNEs in mammals that segregate SNPs between rock-sand cichlid species. 454 Experimentally verified and published genome-wide craniofacial and neural crest 455 enhancers active during early embryonic stages that play a role in shaping the 456 development of neural crest and craniofacial structures in mammals were identified from 457 published literature ${ }^{39,40}$. We used the liftOver tool ${ }^{67}$, which maps orthologous genomic 458 regions between species to convert genomic coordinates from one species to another. 459 Using a Human to Oreochromis niloticus to Metriaclima zebra mapping and a Mouse to 460 Oreochromis niloticus to Metriaclima zebra mapping, we identified the orthologous 461 genomic locations of the published craniofacial and neural crest enhancers in cichlids. 462 We designated any alternately fixed variant (variant with $F_{S T}=1$ ) that was also within an 463 orthologous CNE as putatively involved in the rock-sand divergence (Supplementary 464 Table 2). 
467 We identified 10,391 cichlid genes with human homologues and generated an expression 468 matrix for each gene across 250 human brain structures spanning telencephalon, 469 diencephalon, mesencephalon, and metencephalon using adult human brain microarray 470 data collected by the Allen Brain Institute ${ }^{33}$. Cortical regions and gyri for which fish do 471 not have putative homologues were excluded from the analysis (100/350, leaving 250 472 regions for subsequent analysis). The expression matrix was generated using the 473 get_expression function in the ABAEnrichment Bioconductor package in $\mathrm{R}{ }^{68}$. We then 474 calculated the specificity of expression for each gene in each of these brain regions using 475 the specificity.index function in the $\mathrm{pSI}$ package for $\mathrm{R}$. This function calculates a matrix of 476 gene expression specificity indices, and corresponding $p$-values, as described previously 47769,70 . We then tested whether 1) genes within $25 \mathrm{~kb}$ of rock vs. sand significantly 478 differentiated variants (described above under "Genetically Divergent Regions"), and 2) 479 genes that were differentially expressed between rock- vs. sand-behaving F1 hybrid 480 males, were enriched for transcriptional markers of specific brain regions using the 481 fisher.iteration function with Benjamini-Hochberg correction, again using the pSI package 482 for R. For enrichment testing of differentially expressed genes, we restricted analysis to 483 genes that met the following criteria: 1) transcripts for the gene were detected in all eight 484 paired behaving males, and 2) at least 6 transcripts were detected in each subject.

\section{Staging during gastrulation}

487 Cichlid gastrulation was split into three sub stages within the gastrula stage $9{ }^{71}$. 488 Gastrulation lasts 8 to 12 hours, depending on the species, and is defined as after the 489 shield (as described in zebrafish) stage until the presence of the first somite at the 490 beginning of neurula (stage 10). Embryos were classified as early gastrula (EG) by an 491 asymmetry in epiboly after shield stage until the formation of a ridge that is analogous to 492 the anterior neural ridge (ANR) in chick and mouse and the anterior neural border (ANB) 493 in zebrafish. At that point embryos were classified as mid gastrula (MG). MG lasts until 494 the formation of the dorsal-ventral axis, defined by further lengthening of one side of the 495 embryo, which begins to thicken as epiboly progresses. This is the dorsal side of the 496 embryo, and the side opposite the ANR is classified the ventral side of the embryo. At this 
497 point the embryos are defined as late gastrula (LG). LG ends with the specification of the 498 neural plate, which appears as a portion of the dorsal embryo that is raised relative to 499 ventral side, usually in line with the ANR.

501 Immunohistochemical staining

502 Embryos were harvested at 24 hours post fertilization (hpf) from each of the rock -dwelling 503 cichlids Metriaclima patricki and Metriaclima zebra and the sand-dwelling cichlid 504 Copadichromis borleyi and Tramitichromis intermedius. The embryos were cultured until 505 they reached gastrula stage, approximately 36 to $40 \mathrm{hpf}$, then fixed at intervals throughout 506 gastrula until neurula. The embryos were then treated with auto-fluorescence reducer 507 (1.55mL 5M NaCl, 250ul Tris- $\mathrm{HCl}, \mathrm{pH} 7.5$, and 95mg NaBH4) overnight, and $10 \% 2-$ 508 mercaptoethanol for 1 hour. Next, whole mount in situ hybridization was done, using a 509 modification methods we published previously ${ }^{72}$. ir $\times 1 \mathrm{~b}$ was visualized using Fast Red 510 (naphthol chromogen, Roche Diagnostics), which fluoresces at near red wavelengths 511 (500-650 nm). After in situ hybridization, embryos were immunostained for pSMAD 1,5,8 512 protein, using published protocols ${ }^{73}$. Embryos were then bathed in Vectashield (Vector 513 Labs) containing DAPI and placed in a specially built mold ${ }^{45}$ that accommodates the large 514 yolk and holds the embryo upright. Embryos were then scanned using a Zeiss LSM 700515405 confocal microscope and processed using LSM 700 software and Image J.

Rock-Sand hybridization and genotyping

518 Two rock-sand crosses, one between Copadichromis borleyi (CB, sand-dweller sire) and 519 Metriaclima zebra (MZ, rock-dweller dam) and another between Mchenga conophoros 520 (MC, sand- sire) and Petrotilapia sp. 'thick bar' (PT, rock- dam), were artificially generated 521 by taking the eggs from the dam just prior to spawning and mixing with sperm from the 522 sire. The resultant $F_{1}$ were grown in tanks and allowed to spawn normally to generate $F_{2}$. 523 Several $F_{2}$ broods were taken from multiple $F_{1}$ females for each cross, a total of 355 524 individuals for the $\mathrm{CB} \times \mathrm{MZ}$ cross and 608 for the MC $\times$ PT cross. The embryos were fixed 525 at every stage starting at gastrula (stage 9) until early pharyngula (stage 14). The $F_{2}$ 
526 embryos were RNA-extracted at stage 9. DNA extraction was performed by fixing the 527 embryos (stage 11-14) in 70\% ethanol, then removing the tail from each individual and 528 extracting the DNA using an extraction kit (Qiagen). Following extraction, the $F_{2}$ embryos 529 were genotyped using custom probes (CAAATCTCCC[C/T]CCGCGGC, Taqman custom 530 probes, Invitrogen) designed to identify a SNP in irx1b using RT-PCR. A subset of the 531 embryos was also sequenced at a $900 \mathrm{bp}$ interval around the irx $1 b$ SNP to verify the 532 custom probes.

\section{Quantitative $F_{2}$ Analysis}

535 We quantified $i r \times 1 b$ in $F_{2}$ at stage 9 and separated by genotypic class. The 74 536 heterozygous rock $X$ sand $F_{2}$ embryos were dissected to remove most of the yolk and the 537 total RNA was extracted from each individual using an RNA Extraction Kit (Qiagen).

538 The amount of mRNA specific to each allele of irx $1 b$ was quantified by using the RNA-to539 Ct kit (Invitrogen) and the custom probes. The delta Ct for each heterozygote was 540 generated with the equation, $2^{\wedge}$ (allele from dam - allele from sire). We tested the data 541 with an ANOVA, followed by a Tukey's multiple comparison test to determine significance 542 between genotype classes.

\section{Forebrain and eye measurements}

545 The forebrain and eyes were measured by integrating the area of transverse sections in 546 embryos of rock- and sand-dweller cichlid species, using previously published methods

$547{ }^{21}$. The rock-dweller species included Cynotilapia afra (CA, planktivore), Labeotropheus 548 fuelleborni (LF, algivore) and Metriaclima zebra (MZ, generalist); sand-dweller species 549 included Aulonocara jacobfreibergi (AJ, 'sonar' hunter), Copadichromis borleyi (CB, 550 planktivore) and Mchenga conophoros (MC, insectivore/generalist). Embryos from each 551 species, as well as the $F_{2}$ individuals, were measured starting from the earliest the 552 telencephalon can be differentiated from the forebrain (mid-somitogenesis, stage 12) and 553 at each subsequent stage until the forebrain has defined prosomeres (early pharyngula, 554 stage 14) ${ }^{22}$. To keep measurements standardized across stages, all measurements were 
555 defined by forebrain morphology at the earliest timepoint (stage 12). The 'eye' 556 measurement remains consistent at all stages, the 'anterior' measurement includes the 557 telencephalon and presumptive olfactory bulb, and the 'posterior' measurement includes 558 the diencephalon and each of its constitutive prosomeres (dorsal and ventral thalamus 559 and hypothalamus). To facilitate measurements, we used gene expression of $r \times 3$ (for 560 stage 12 embryos) and pax6 (stage 13 and 14) to identify the different structures of the 561 forebrain and eye.

RNA Extraction and Sequencing, Adult Social Behavior

Two adult $F_{1}$ hybrid males from rock- $x$ sand- interspecific crosses (Supplementary table 5) were introduced to an assay tank containing 3-5 hybrid females of the same cross with simulated rock habitat on one side and simulated sand habitat on the other side separated by empty tank space (Figure $3 \mathrm{~A}$ ). Males were observed over a period of four days for courtship behavior, either similar to a rock parent (courting females over the rocky parts of the tank), here designated "social rock" --- or to a sand parent (bower building in the sand side of the tank while courting females), here designated "social sand." When courtship behavior of both males was observed, we primed experiments the next morning by resetting the tanks -- knocking down or filling in the sand bower, and adjusting the rock

574 habitat. Both males were allowed to exhibit courtship behavior over a period of 90

575 minutes. Males were sacrificed immediately after this 90 -minute period by rapid 576 decapitation and whole brains were immediately stored in RNAlater (Thermo Fisher Cat\# 577 AM7020) within 20 minutes of decapitation. Two tanks with one $F_{1}$ male and 3-5 $F_{1}$ 578 females were also observed. These males exhibited social rock behavior and were 579 designated as "lone" in the behavior assay; their brains were collected in the exact same 580 way as the other samples.

582 Tissues were frozen in liquid nitrogen, homogenized using a mortar and pestle and placed 583 in trizol. Following standard chloroform extraction, RNeasy mini columns (Qiagen Cat 584 No./ID: 74104) were utilized to purify RNA for sequencing. Total RNA was quantified 
585 using Qubit (Molecular Probes) and quality analyzed using the Agilent 2100 Bioanalyzer

586 System for RNA library preparation. RNA input was normalized to $1 \mu \mathrm{g}$ and libraries were 587 prepared using the TruSeq Stranded mRNA Sample Prep Kit (Illumina- Kit A). Libraries 588 were again quantified, quality assessed, and normalized for sequencing on the HiSeq 5892500 Illumina Sequencing System (Georgia Tech Genomics Core, standard practices). 590 Experimental design and raw files can be accessed on the NCBI Gene Expression 591 Omnibus database under the accession number GSE122500.

\section{Differential Gene Expression Analysis}

594 Raw sequence reads from whole brain transcriptomes were quality controlled using the 595 NGS QC Toolkit ${ }^{74}$. Raw reads with an average PHRED quality score below 20 were 596 filtered out. Filtered reads were also trimmed of low-quality bases at the 3' end. High 597 quality sequence reads were aligned to the M.zebra reference genome 598 MZ_UMD2a ${ }^{30}$ using TopHat v2.0.9 ${ }^{75}$. On average, across all samples, over $95 \%$ of reads 599 mapped to the reference genome. The resulting TopHat2 output bam files were sorted 600 and converted to sam files using samtools v0.19 ${ }^{76}$. Sorted sam files were used as input 601 for the HTSeq-count v0.6.1 program to obtain fragment counts for each locus 77 . 602 Fragment counts were scale-normalized across all samples using the calcNormFactors 603 function in the edgeR package v3.6.8 ${ }^{78}$. Relative consistency among replicates and 604 samples was determined via the Multidimensional scaling (MDS) feature within the edgeR 605 package in $\mathrm{R}$. The native $\mathrm{R}$ function hclust(dist) used to cluster samples. Scale606 normalized fragment counts were converted into $\log _{2}$ counts per million reads mapped 607 (cpm) with precision weights using voom and fit to a linear model using limma v3.20.9 ${ }^{79}$. 608 Pairwise contrasts were constructed between socially rock and socially sand samples. 609 After correcting for multiple comparisons using the Benjamini-Hochberg method ${ }^{80}$, genes 610 were considered differentially expressed between socially rock and socially sand samples

611 if they exhibited both a fold change $\geq 2$ and $P_{a d j}<0.05$. Using Treefam based mapping 61

612 the cichlid gene names were converted to human analogs and functional enrichment was 613 determined using the TOPPFUN web-browser ${ }^{62}$. 


\section{Ethics Statement}

615 All of the animals were handled according to methods approved by the Georgia Tech 616 Institutional Animal Care and Use Committee (IACUC) protocols (permit A100029) with 617 every effort made to minimize suffering and is in compliance with the ARRIVE guidelines. 
618 Acknowledgements: This work was supported by grants from the NIH (R01GM101095, 619 2R01DE019637-10 to JTS and F32GM128346-01A1 to ZVJ) and the Human Frontiers 620 Science Program (RGP0052/2019 to JTS). We would like to thank Shweta Biliya and the 621 Genomics Core at Georgia Tech for invaluable assistance with the NGS sequencing. We 622 also thank the members of the Streelman lab for comments on this manuscript. 
6251 Boyle, E. A., Li, Y. I. \& Pritchard, J. K. An Expanded View of Complex Traits: From

626 Polygenic to Omnigenic. Cell 169, 1177-1186, doi:10.1016/j.cell.2017.05.038 (2017).

6272 Fields, C., Adams, M. D., White, O. \& Venter, J. C. How many genes in the human 628 genome? Nature Genetics 7, 345-346, doi:10.1038/ng0794-345 (1994).

6293 Maurano, M. T. et al. Systematic localization of common disease-associated variation in 630 regulatory DNA. Science, 9718-9723 (2012).

6314 Degner, J. F. et al. DNase I sensitivity QTLs are a major determinant of human expression $632 \quad$ variation. Nature 482, 390 (2012).

6335 Streelman, J., Peichel, C. L. \& Parichy, D. Developmental genetics of adaptation in fishes: 634 the case for novelty. Annu. Rev. Ecol. Evol. Syst. 38, 655-681 (2007).

6356 Colosimo, P. F. et al. The genetic architecture of parallel armor plate reduction in 636 threespine sticklebacks. Plos Biol 2, E109, doi:10.1371/journal.pbio.0020109 (2004).

6377 Kratochwil, C. F. et al. Agouti-related peptide 2 facilitates convergent evolution of stripe 638 patterns across cichlid fish radiations. Science 362, 457-460, doi:10.1126/science.aao6809 639 (2018).

6408 Albertson, R. C., Streelman, J. T., Kocher, T. D. \& Yelick, P. C. Integration and evolution of the cichlid mandible: The molecular basis of alternate feeding strategies. $P$ Natl Acad Sci USA 102, 16287-16292, doi:DOI 10.1073/pnas.0506649102 (2005).

6439 Shapiro, M. D. et al. Genomic diversity and evolution of the head crest in the rock pigeon. $644 \quad$ Science 339, 1063-1067, doi:10.1126/science.1230422 (2013).

$64510 \quad$ Lamichhaney, S. et al. Evolution of Darwin's finches and their beaks revealed by genome 646 sequencing. Nature 518, 371-375 (2015).

64711 Okhovat, M., Berrio, A., Wallace, G., Ophir, A. G. \& Phelps, S. M. Sexual fidelity trade648 offs promote regulatory variation in the prairie vole brain. Science 350, 1371-1374 (2015).

64912 Bendesky, A. et al. The genetic basis of parental care evolution in monogamous mice. $650 \quad$ Nature 544, 434 (2017).

65113 Pfenning, A. R. et al. Convergent transcriptional specializations in the brains of humans $652 \quad$ and song-learning birds. Science 346, 1256846 (2014). 
65314 Greenwood, A. K., Wark, A. R., Yoshida, K. \& Peichel, C. L. Genetic and neural 654 modularity underlie the evolution of schooling behavior in threespine sticklebacks. Curr 655 Biol 23, 1884-1888, doi:10.1016/j.cub.2013.07.058 (2013).

65615 Kocher, T. D. Adaptive evolution and explosive speciation: The cichlid fish model. Nat 657 Rev Genet 5, 288-298 (2004).

65816 Loh, Y. H. E. et al. Comparative analysis reveals signatures of differentiation amid 659 genomic polymorphism in Lake Malawi cichlids. Genome Biology 9 (2008).

66017 Loh, Y. H. E. et al. Origins of Shared Genetic Variation in African Cichlids. Mol Biol Evol 661 30, 906-917 (2013).

66218 Streelman, J. T., Albertson, R. C. \& Kocher, T. D. Genome mapping of the orange blotch 663 colour pattern in cichlid fishes. Mol Ecol 12, 2465-2471, doi:10.1046/j.1365664 294x.2003.01920.x (2003).

66519 Roberts, R. B., Ser, J. R. \& Kocher, T. D. Sexual conflict resolved by invasion of a novel 666 sex determiner in Lake Malawi cichlid fishes. Science 326, 998-1001 (2009).

Parnell, N. F. \& Streelman, J. T. Genetic interactions controlling sex and color establish the potential for sexual conflict in Lake Malawi cichlid fishes. Heredity 110, 239-246 (2013).

Sylvester, J. B. et al. Competing signals drive telencephalon diversity. Nat Commun 4, 4 (2013).

Sylvester, J. B. et al. Brain diversity evolves via differences in patterning. Proceedings of the National Academy of Sciences 107, 9718-9723 (2010).

Baran, N. M. \& Streelman, J. T. Ecotype differences in aggression, neural activity and behaviorally relevant gene expression in cichlid fish. Genes Brain Behav 19, e12657, doi:10.1111/gbb.12657 (2020).

York, R. A. et al. Behavior-dependent cis regulation reveals genes and pathways associated with bower building in cichlid fishes. Proceedings of the National Academy of Sciences 115, E11081-e11090, doi:10.1073/pnas.1810140115 (2018).

Johnson, Z. V. et al. Exploratory behaviour is associated with microhabitat and evolutionary radiation in Lake Malawi cichlids. Animal Behaviour 160, 121-134, doi:https://doi.org/10.1016/j.anbehav.2019.11.006 (2020).

68326 Fraser, G. J. et al. An ancient gene network is co-opted for teeth on old and new jaws. Plos $684 \quad$ Biol 7, e1000031 (2009).

68527 Streelman, J. T. \& Danley, P. D. The stages of vertebrate evolutionary radiation. Trends 686 Ecol Evol 18, 126-131 (2003). 
Malinsky, M. et al. Whole-genome sequences of Malawi cichlids reveal multiple radiations interconnected by gene flow. Nat Ecol Evol 2, 1940-1955, doi:10.1038/s41559-018-0717-

29 McKaye, K. R., Louda, S. M. \& Jay R. Stauffer, J. Bower Size and Male Reproductive Success in a Cichlid Fish Lek. The American Naturalist 135, 597-613, doi:10.1086/285064 (1990).

Conte, M. A. et al. Chromosome-scale assemblies reveal the structural evolution of African cichlid genomes. GigaScience 8, doi:10.1093/gigascience/giz030 (2019).

Brawand, D. et al. The genomic substrate for adaptive radiation in African cichlid fish. Nature 513, 375-381 (2014).

Ben-Ari Fuchs, S. et al. GeneAnalytics: An Integrative Gene Set Analysis Tool for Next Generation Sequencing, RNAseq and Microarray Data. Omics 20, 139-151 (2016).

Hawrylycz, M. J. et al. An anatomically comprehensive atlas of the adult human brain transcriptome. Nature 489, 391-399, doi:10.1038/nature11405 (2012).

Adhikari, A. et al. Basomedial amygdala mediates top-down control of anxiety and fear. Nature 527, 179-185, doi:10.1038/nature15698 (2015).

Mesquita, L. T. et al. New insights on amygdala: Basomedial amygdala regulates the physiological response to social novelty. Neuroscience 330, 181-190, doi:https://doi.org/10.1016/j.neuroscience.2016.05.053 (2016).

Carvalho, F. R., Nóbrega, C. D. R. \& Martins, A. T. Mapping gene expression in social anxiety reveals the main brain structures involved in this disorder. Behavioural Brain Research 394, 112808, doi:https://doi.org/10.1016/j.bbr.2020.112808 (2020).

Abrahams, B. S. et al. SFARI Gene 2.0: a community-driven knowledgebase for the autism spectrum disorders (ASDs). Mol Autism 4, 36, doi:10.1186/2040-2392-4-36 (2013).

Piñero, J. et al. DisGeNET: a comprehensive platform integrating information on human disease-associated genes and variants. Nucleic Acids Research 45, D833-D839, doi:10.1093/nar/gkw943 (2017).

Attanasio, C. et al. Fine Tuning of Craniofacial Morphology by Distant-Acting Enhancers. Science 342 (2013).

Rada-Iglesias, A. et al. Epigenomic Annotation of Enhancers Predicts Transcriptional Regulators of Human Neural Crest. Cell Stem Cell 11, 633-648 (2012).

41 Simoes-Costa, M. \& Bronner, M. E. Establishing neural crest identity: a gene regulatory recipe. Development 142, 242-257, doi:10.1242/dev.105445 (2015). 
42 Roberts, R. B., Hu, Y., Albertson, R. C. \& Kocher, T. D. Craniofacial divergence and ongoing adaptation via the hedgehog pathway. Proc Natl Acad Sci U S A 108, 1319413199, doi:10.1073/pnas.1018456108 (2011).

43 Cavodeassi, F., Modolell, J. \& Gómez-Skarmeta, J. L. The Iroquois family of genes: from body building to neural patterning. Development 128, 2847-2855 (2001).

4 Bielen, H. \& Houart, C. BMP signaling protects telencephalic fate by repressing eye identity and its Cxcr4-dependent morphogenesis. Developmental cell 23, 812-822 (2012).

45 White, D. E. et al. Quantitative multivariate analysis of dynamic multicellular morphogenic trajectories. Integrative Biology 7, 825-833, doi:10.1039/c5ib00072f (2015).

46 Volff, J. N., Korting, C. \& Schartl, M. Multiple lineages of the non-LTR retrotransposon Rex1 with varying success in invading fish genomes. Mol Biol Evol 17, 1673-1684, doi:10.1093/oxfordjournals.molbev.a026266 (2000).

47 Olton, D. S., Becker, J. T. \& Handelmann, G. E. Hippocampus, space, and memory. Behavioral and Brain sciences 2, 313-322 (1979).

48 Hitti, F. L. \& Siegelbaum, S. A. The hippocampal CA2 region is essential for social memory. Nature 508, 88-92 (2014).

49 Chiang, M.-C., Huang, A. J., Wintzer, M. E., Ohshima, T. \& McHugh, T. J. A role for CA3 in social recognition memory. Behavioural brain research 354, 22-30 (2018).

50 Zou, D. et al. DREADD in parvalbumin interneurons of the dentate gyrus modulates anxiety, social interaction and memory extinction. Current Molecular Medicine 16, 91-102 (2016).

51 O’Connell, L. A. \& Hofmann, H. A. Evolution of a vertebrate social decision-making network. Science 336, 1154-1157 (2012).

52 McLean, C. Y. et al. Human-specific loss of regulatory DNA and the evolution of humanspecific traits. Nature 471, 216-219, doi:10.1038/nature09774 (2011).

53 Finlay, B. L. \& Darlington, R. B. Linked regularities in the development and evolution of mammalian brains. Science 268, 1578-1584, doi:10.1126/science.7777856 (1995).

54 Brenner, S. Genetics of Caenorhabditis-Elegans. Genetics 77, 71-94 (1974).

55 Fernald, R. D. \& Maruska, K. P. Social information changes the brain. Proc Natl Acad Sci U S A 109 Suppl 2, 17194-17199, doi:10.1073/pnas.1202552109 (2012).

$56 \mathrm{Li}, \mathrm{H}$. \& Durbin, R. Fast and accurate short read alignment with Burrows-Wheeler transform. Bioinformatics 25, 1754-1760, doi:10.1093/bioinformatics/btp324 (2009). 
57 Van der Auwera, G. A. et al. From FastQ data to high confidence variant calls: the Genome Analysis Toolkit best practices pipeline. Current protocols in bioinformatics 43, 11.10.1133, doi:10.1002/0471250953.bi1110s43 (2013).

58 Lee, T. H., Guo, H., Wang, X. Y., Kim, C. \& Paterson, A. H. SNPhylo: a pipeline to construct a phylogenetic tree from huge SNP data. Bmc Genomics 15 (2014).

59 Danecek, P. et al. The variant call format and VCFtools. Bioinformatics 27, 2156-2158 (2011).

60 Cingolani, P. et al. A program for annotating and predicting the effects of single nucleotide polymorphisms, SnpEff: SNPs in the genome of Drosophila melanogaster strain w(1118); iso-2; iso-3. Fly 6, 80-92 (2012).

61 Ramakrishnan Varadarajan, A., Mopuri, R., Streelman, J. T. \& McGrath, P. T. Genomewide protein phylogenies for four African cichlid species. BMC Evol Biol 18, 1, doi:10.1186/s12862-017-1072-2 (2018).

62 Chen, J., Bardes, E. E., Aronow, B. J. \& Jegga, A. G. ToppGene Suite for gene list enrichment analysis and candidate gene prioritization. Nucleic Acids Res 37, W305-W311, doi:10.1093/nar/gkp427 (2009).

63 Harris, R. S. Improved Pairwise Alignment of Genomic DNA Doctor of Philosophy thesis, The Pennsylvania State University, (2007).

64 Blanchette, M. et al. Aligning multiple genomic sequences with the threaded blockset aligner. Genome research 14, 708-715 (2004).

65 Siepel, A. et al. Evolutionarily conserved elements in vertebrate, insect, worm, and yeast genomes. Genome Res 15, 1034-1050, doi:10.1101/gr.3715005 (2005).

66 Siepel, A. \& Haussler, D. Phylogenetic estimation of context-dependent substitution rates by maximum likelihood. Mol Biol Evol 21, 468-488 (2004).

67 Kent, W. J. et al. The human genome browser at UCSC. Genome research 12, 996-1006 (2002).

68 Grote, S., Prufer, K., Kelso, J. \& Dannemann, M. ABAEnrichment: an R package to test for gene set expression enrichment in the adult and developing human brain. Bioinformatics 32, 3201-3203, doi:10.1093/bioinformatics/btw392 (2016).

69 Dougherty, J. D., Schmidt, E. F., Nakajima, M. \& Heintz, N. Analytical approaches to RNA profiling data for the identification of genes enriched in specific cells. Nucleic Acids Res 38, 4218-4230, doi:10.1093/nar/gkq130 (2010).

70 Xu, X., Wells, A. B., O'Brien, D. R., Nehorai, A. \& Dougherty, J. D. Cell type-specific expression analysis to identify putative cellular mechanisms for neurogenetic disorders. $J$ Neurosci 34, 1420-1431, doi:10.1523/JNEUROSCI.4488-13.2014 (2014). 
Murata, Y. et al. Allometric growth of the trunk leads to the rostral shift of the pelvic fin in teleost fishes. Dev Biol 347, 236-245, doi:10.1016/j.ydbio.2010.07.034 (2010).

78972 Fraser, G. J., Bloomquist, R. F. \& Streelman, J. T. A periodic pattern generator for dental 790 diversity. Bmc Biol 6 (2008).

79173 Tucker, J. A., Mintzer, K. A. \& Mullins, M. C. The BMP signaling gradient patterns dorsoventral tissues in a temporally progressive manner along the anteroposterior axis. Dev Cell 14, 108-119, doi:10.1016/j.devcel.2007.11.004 (2008).

Patel, R. K. \& Jain, M. NGS QC Toolkit: A Toolkit for Quality Control of Next Generation Sequencing Data. PLoS One 7, 7 (2012).

Trapnell, C., Pachter, L. \& Salzberg, S. L. TopHat: discovering splice junctions with RNASeq. Bioinformatics 25, 1105-1111 (2009).

Li, H. et al. The Sequence Alignment/Map format and SAMtools. Bioinformatics 25, 20782079 (2009).

Anders, S., Pyl, P. T. \& Huber, W. HTSeq--a Python framework to work with highthroughput sequencing data. Bioinformatics 31, 166-169 (2015). differential expression analysis of digital gene expression data. Bioinformatics 26, 139-140 (2010).

Ritchie, M. E. et al. limma powers differential expression analyses for RNA-sequencing and microarray studies. Nucleic Acids Res 43, e47 (2015).

Hochberg, Y. \& Benjamini, Y. More powerful procedures for multiple significance testing. Stat Med 9, 811-818 (1990). compendium of developmental gene expression in Lake Malawi cichlid fishes. BMC Dev Biol 17, 3, doi:10.1186/s12861-017-0146-0 (2017). 
819 Author Contributions: CP, JBS, KA and JTS designed research; CP, JBS, KA, MWN, KP, RFB

820 performed research; CP, KA, MM, ZVJ and PTM analyzed data; CP, JBS, ZVJ and JTS wrote the paper.

821

822 Competing Interest Statement: The Authors declare no competing interests.

823 


\section{Figures:}

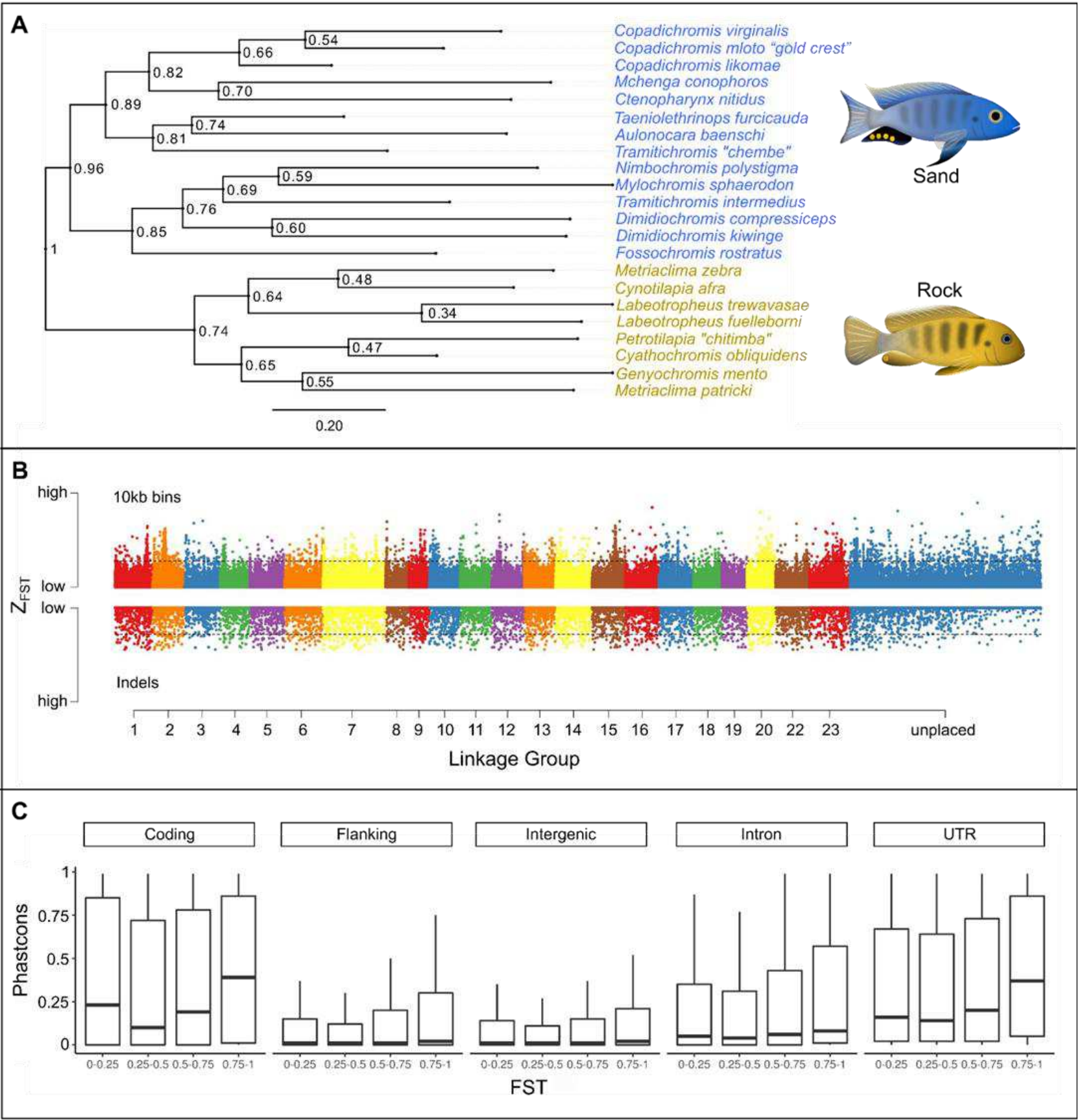

826 Figure 1: The genomic substrate for rock vs. sand evolution | (A) A maximum likelihood

827 phylogeny of eight rock- and fourteen sand-dwelling species, based on variable sites

828 (informative SNP and InDels) identified throughout the genome. "Sand" species contain

829 representatives of the lineages "shallow benthic, deep benthic and utaka" from ${ }^{28}$, while

830 the "rock" species correspond to the "mbuna" lineage. (B) A plot of Z-FST (Fst normalized

831 using Fisher's Z-transformation) across the genome, plotting genomic divergence 
832 between rock- vs. sand-dwelling groups. Single nucleotide polymorphisms (SNPs) 833 summed over $10 \mathrm{~kb}$ bins and insertion-deletion mutations (InDels) are shown on the same 834 scale. Numbers along the $x$-axis refer to linkage groups (i.e., chromosomes) and 835 threshold lines indicate 2.5\% FDR. (C) Evolutionary conservation (PhastCons) scores 836 were calculated for each nucleotide across the genome, subdivided by genome 837 annotation and plotted by bins of increasing FST. The PhastCons score for each genome 838 category is significantly higher for increasing bins of FST (Wilcoxon rank sum $p$ value < 839 2e-16). 


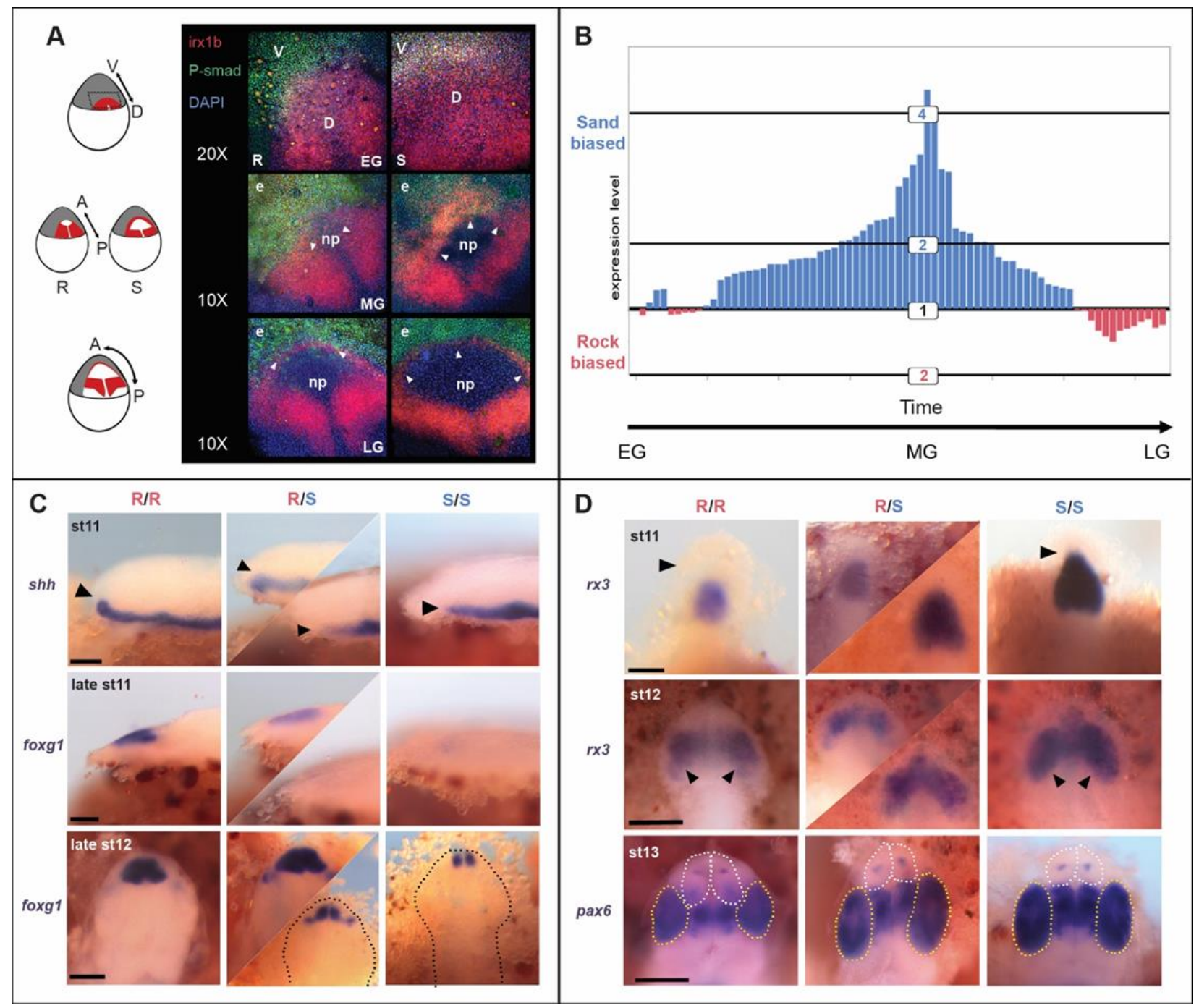

842 Figure 2: A gastrula-stage map of forebrain diversification | (A) Double in situ hybridization

843 - immunohistochemistry to visualize irx1b expression (red) and BMP [PSMAD] activity

844 (green) across the stages of cichlid gastrulation; DAPI in blue. Three rows represent early,

845 mid and late gastrulation (EG, MG, LG) in embryos of rock- (R) and sand-dwelling (S)

846 species. Sand-dwellers show expanded irx1 expression in the dorsal portion of the

847 embryo at EG, expanded irx1b expression in the anterior domain at MG (arrowheads)

848 and clear PSMAD activity from the developing neural plate (np) earlier in LG

849 (arrowheads). e=epidermis. Schematics at left show irx1b expression domains in red, on

850 cartoons of cichlid embryos. (B) Relative expression of rock- (red) and sand- (blue) irx1b

851 alleles, sampled from 74 heterozygous rock $X$ sand $F_{2}$ embryos, across the stages of

852 gastrulation. $F_{2}$ embryos were sampled at stage 9 (gastrulation). Because Malawi cichlid

853 species are maternal mouthbrooders and eggs are fertilized in batches per brood, each 
854 brood's embryos vary in timing of fertilization by up to $4 \mathrm{~h}$. Embryos within broods can 855 therefore be sub-staged in gastrulation (see methods). Each bar on the plot represents 856 the relative allelic expression of sand- and rock- irx $1 b$ in a heterozygous $F_{2}$ individual. 857 Quantification of allele-specific expression (ASE) shows that levels are sand-biased, and 858 that this effect is strongest in MG. (C) in situ hybridization of shh and foxg1, during neurula 859 stages, showing development of the telencephalon in rock- $X$ sand- $F_{2}$ embryos, indexed 860 for irx $1 b$ genotype. $F_{2}$ individuals homozygous for rock- $i r x 1 b$ alleles $(R / R)$ show a more 861 dorsal progression of shh expression (black arrowheads), an earlier and a larger 862 expression domain of the telencephalon marker foxg1. The top two rows are lateral views; 863 bottom row is a dorsal view. Dotted lines demarcate the outline of the embryo in dorsal 864 view. Heterozygous individuals exhibit greater variation in expression domains (middle 865 columns), indicating that genetic factors other than variants in irx $1 b$ contribute to this 866 phenotype. (D) in situ hybridization for $r \times 3$ and pax6, during neurula stages, chart the 867 development of the eye field in rock- $X$ sand- $F_{2}$ embryos indexed for irx $1 b$ genotypes. $F_{2}$ 868 individuals homozygous for sand-irx $1 b$ alleles (S/S) show larger domains of $r \times 3$ (black 869 arrowheads) and larger eyes (pax6, also marked by yellow dotted line), but smaller 870 telencephala (white dotted line). All panels are dorsal views. Heterozygous individuals 871 exhibit greater variation in expression domains (middle columns), indicating that genetic 872 factors other than variants in irx $1 b$ contribute to this phenotype. 


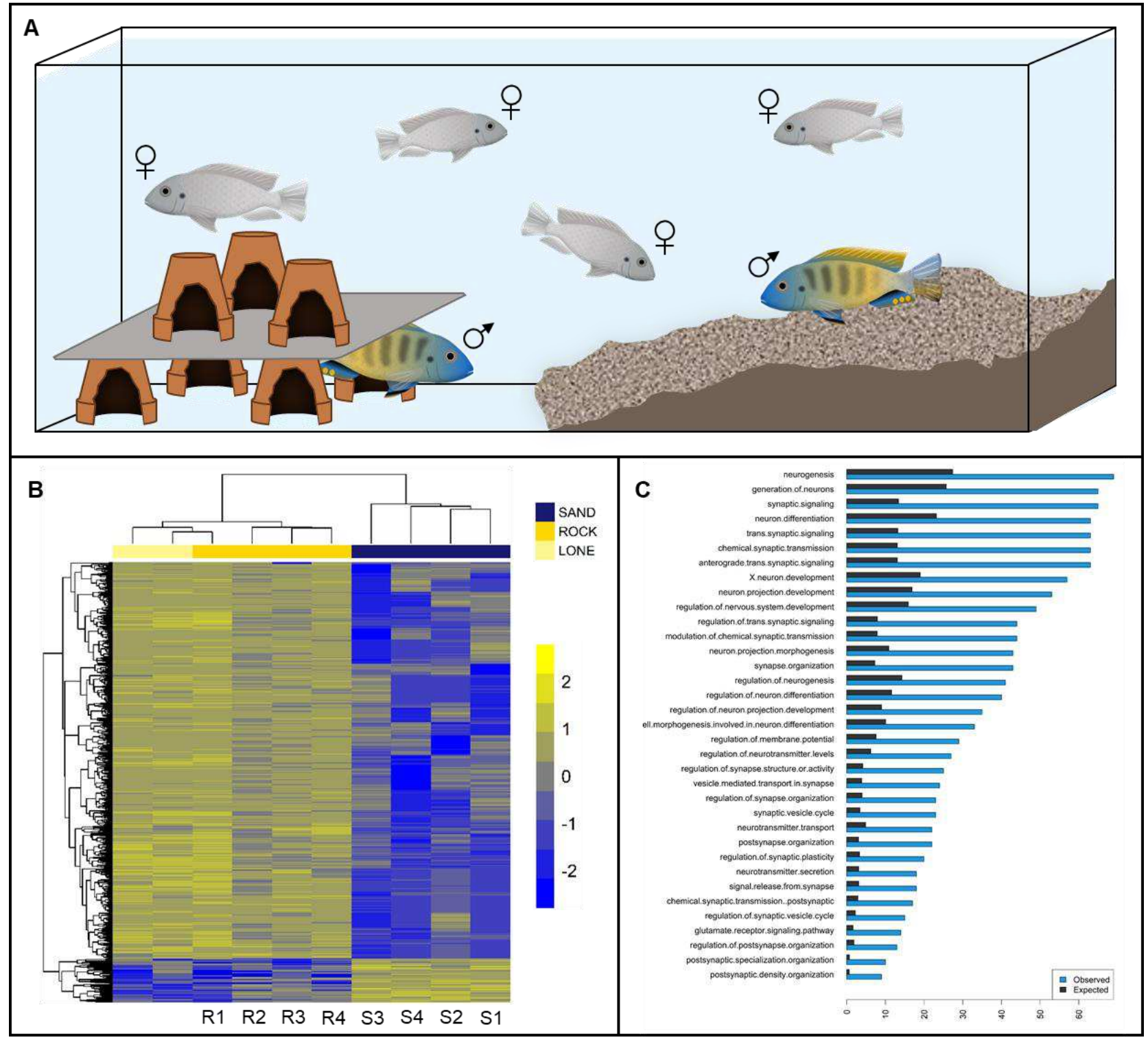

875 Figure 3: Genomics of divergent social context | (A) Schematic of the social context 876 behavioral paradigm, in which rock-, sand- and rock- $X$ sand- $F_{1}$ hybrids were evaluated.

877 (B) Heatmap of genes differentially expressed in the brains of $F_{1}$ males behaving in either 878 rock or sand social contexts. Each row in the heatmap is a gene, each column is an 879 individual. $R$ individuals are socially rock, $S$ individuals are socially sand and the numbers 880 indicate sibling pairs. Two $F_{1}$ males were not paired with other males, and courted 881 females over the rock habitat (lone). All other $F_{1}$ males $(n=8)$ were introduced to the 882 testing arena in dyads. Notably, male brain gene expression clusters by social context 883 and not fraternal relationships. (C) Gene Ontology (GO) Biological Process terms that 
884 show greater than expected overlap between (i) genes differentially expressed in the 885 brains of social rock- vs. social sand- males and (ii) genes that are differentiated in the 886 genomes of rock- vs sand- species groups. 


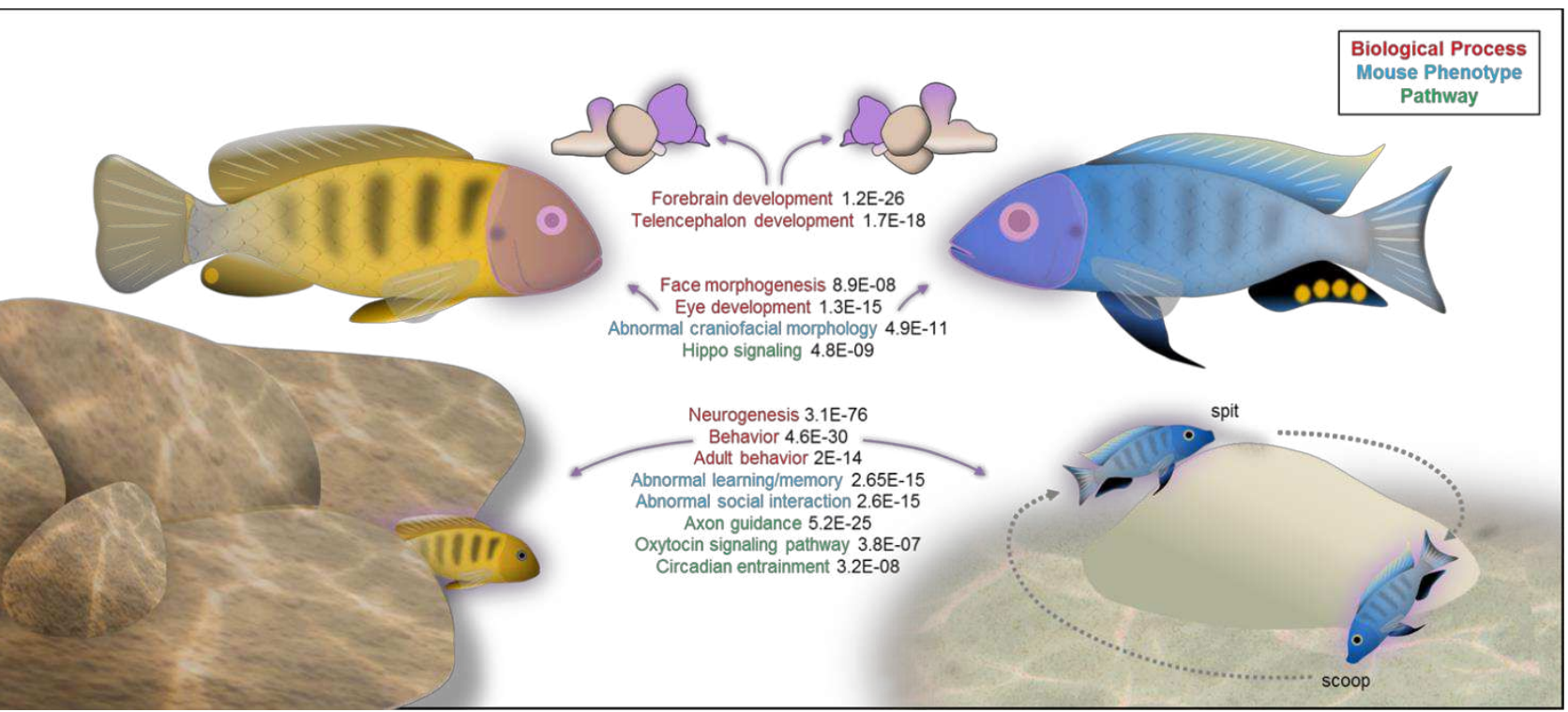

889 Figure 4: Genome-enabled discovery of evolutionary change in morphology and behavior.

890 | Summary cartoon synthesizing significant enrichment categories that differentiate the 891 genomes of rock- vs. sand-dwelling Malawi cichlids. Strong and consistent enrichment of 892 craniofacial, neural and behavioral categories motivated follow-on experiments in early 893 brain development (Figure 2) and adult social behavior (Figure 3). 


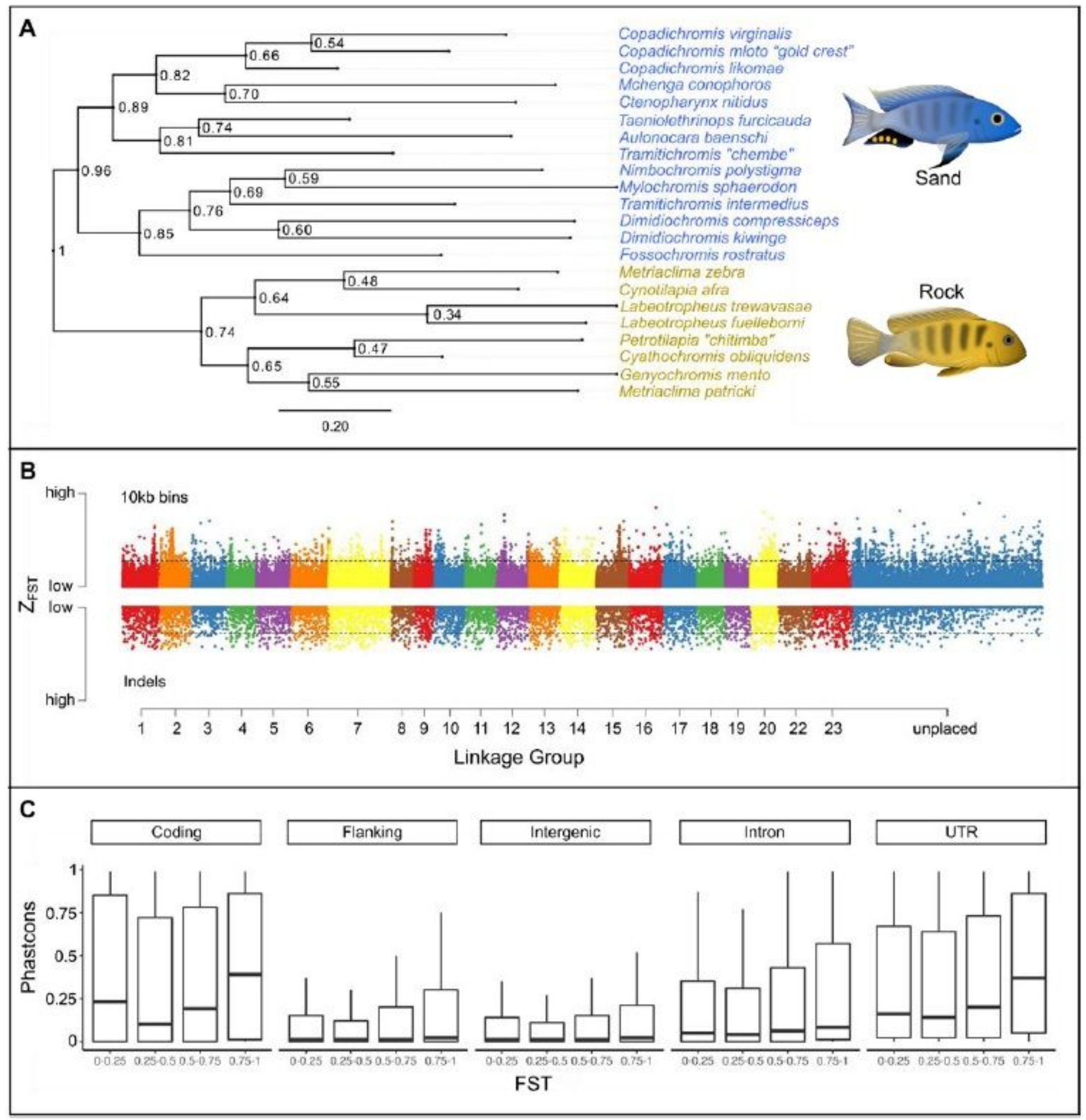

\section{Figure 1}

The genomic substrate for rock vs. sand evolution I (A) A maximum likelihood phylogeny of eight rockand fourteen sand-dwelling species, based on variable sites(informative SNP and InDels) identified throughout the genome. "Sand" species contain representatives of the lineages "shallow benthic, deep 
benthic and utaka" from 28, while the "rock" species correspond to the "mbuna" lineage. (B) A plot of ZFST (FST normalized using Fisher's Z-transformation) across the genome, plotting genomic divergence between rock-vs. sand-dwelling groups. Single nucleotide polymorphisms (SNPs) summed over 10kb bins and insertion-deletion mutations (InDels) are shown on the same scale. Numbers along the $x$-axis refer to linkage groups (i.e., chromosomes) and threshold lines indicate 2.5\% FDR. (C) Evolutionary conservation (PhastCons) scores were calculated for each nucleotide across the genome, subdivided by genome annotation and plotted by bins of increasing FST. The PhastCons score for each genome category is significantly higher for increasing bins of FST (Wilcoxon rank sum p value < 2e-16).

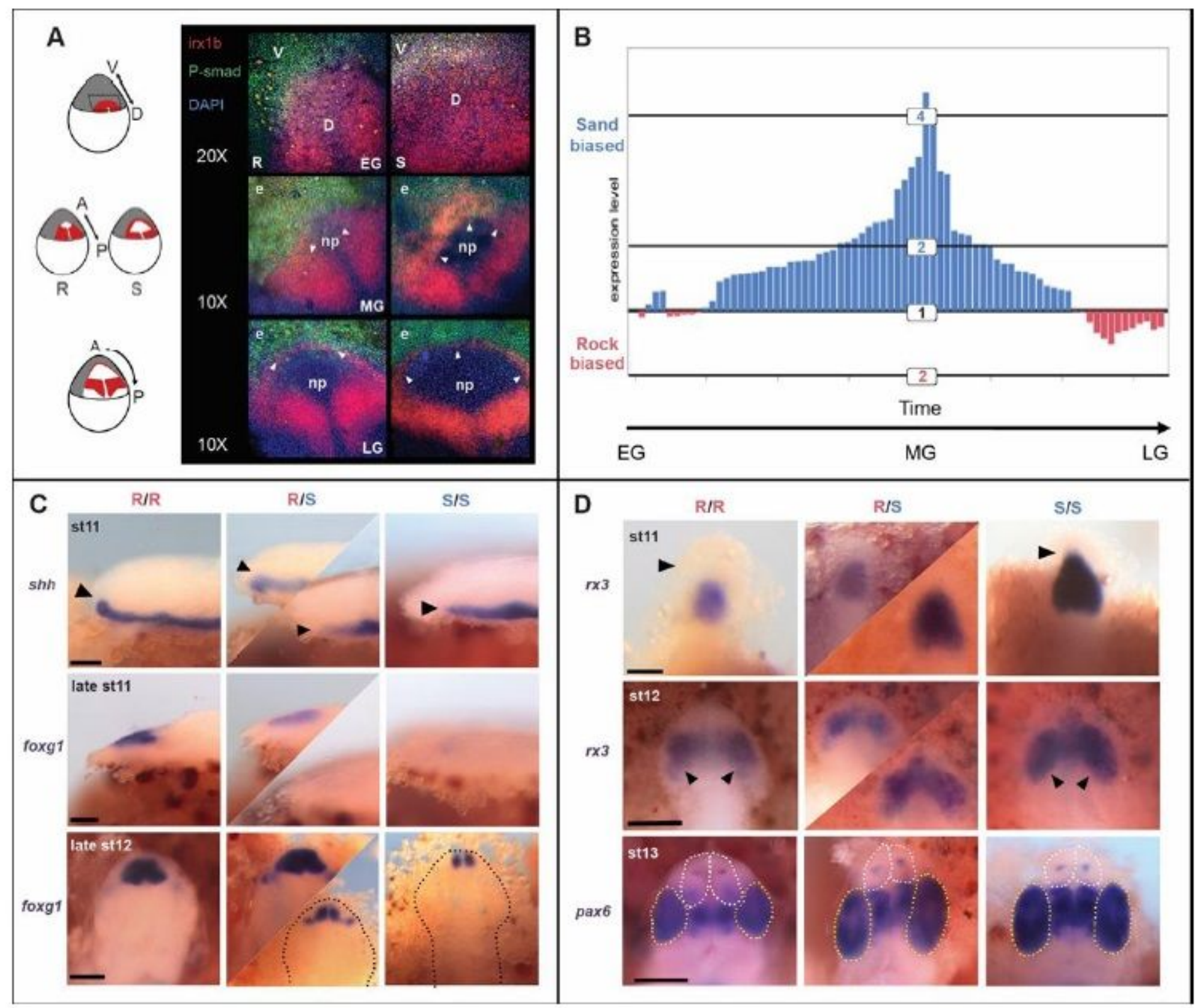

\section{Figure 2}

A gastrula-stage map of forebrain diversification I (A) Double in situ hybridization immunohistochemistry to visualize irx1b expression (red) and BMP [PSMAD] activity 8(green) across the stages of cichlid gastrulation; DAPI in blue. Three rows represent early, mid and late gastrulation (EG, MG, 
LG) in embryos of rock- (R) and sand-dwelling (S) species. Sand-dwellers show expanded irx1 expression in the dorsal portion of the embryo at EG, expanded irx1b expression in the anterior domain at MG (arrowheads) and clear PSMAD activity from the developing neural plate (np) earlier in LG (arrowheads). $\mathrm{e}=$ epidermis. Schematics at left show irx1b expression domains in red, on cartoons of cichlid embryos. (B) Relative expression of rock- (red) and sand- (blue) irx $1 \mathrm{~b}$ alleles, sampled from 74 heterozygous rock $X$ sand F2 embryos, across the stages of gastrulation. F2 embryos were sampled at stage 9 (gastrulation). Because Malawi cichlid species are maternal mouthbrooders and eggs are fertilized in batches per brood, each brood's embryos vary in timing of fertilization by up to $4 \mathrm{~h}$. Embryos within broods can therefore be sub-staged in gastrulation (see methods). Each bar on the plot represents the relative allelic expression of sand- and rock- irx $1 \mathrm{~b}$ in a heterozygous F2 individual. Quantification of allele-specific expression (ASE) shows that levels are sand-biased, and that this effect is strongest in MG. (C) in situ hybridization of shh and foxg1, during neurula stages, showing development of the telencephalon in rock- $X$ sand- $F 2$ embryos, indexed for irx1b genotype. $F 2$ individuals homozygous for rock- irx $1 b$ alleles (R/R) show a more dorsal progression of shh expression (black arrowheads), an earlier and a larger expression domain of the telencephalon marker foxg1. The top two rows are lateral views; bottom row is a dorsal view. Dotted lines demarcate the outline of the embryo in dorsal view. Heterozygous individuals exhibit greater variation in expression domains (middle columns), indicating that genetic factors other than variants in irx $1 \mathrm{~b}$ contribute to this phenotype. (D) in situ hybridization for rx3 and pax6, during neurula stages, chart the development of the eye field in rock- $X$ sand- $F 2$ embryos indexed for irx $1 \mathrm{~b}$ genotypes. F2 individuals homozygous for sand- irx $1 \mathrm{~b}$ alleles (S/S) show larger domains of rx3 (black arrowheads) and larger eyes (pax6, also marked by yellow dotted line), but smaller telencephala (white dotted line). All panels are dorsal views. Heterozygous individuals exhibit greater variation in expression domains (middle columns), indicating that genetic factors other than variants in irx $1 \mathrm{~b}$ contribute to this phenotype. 


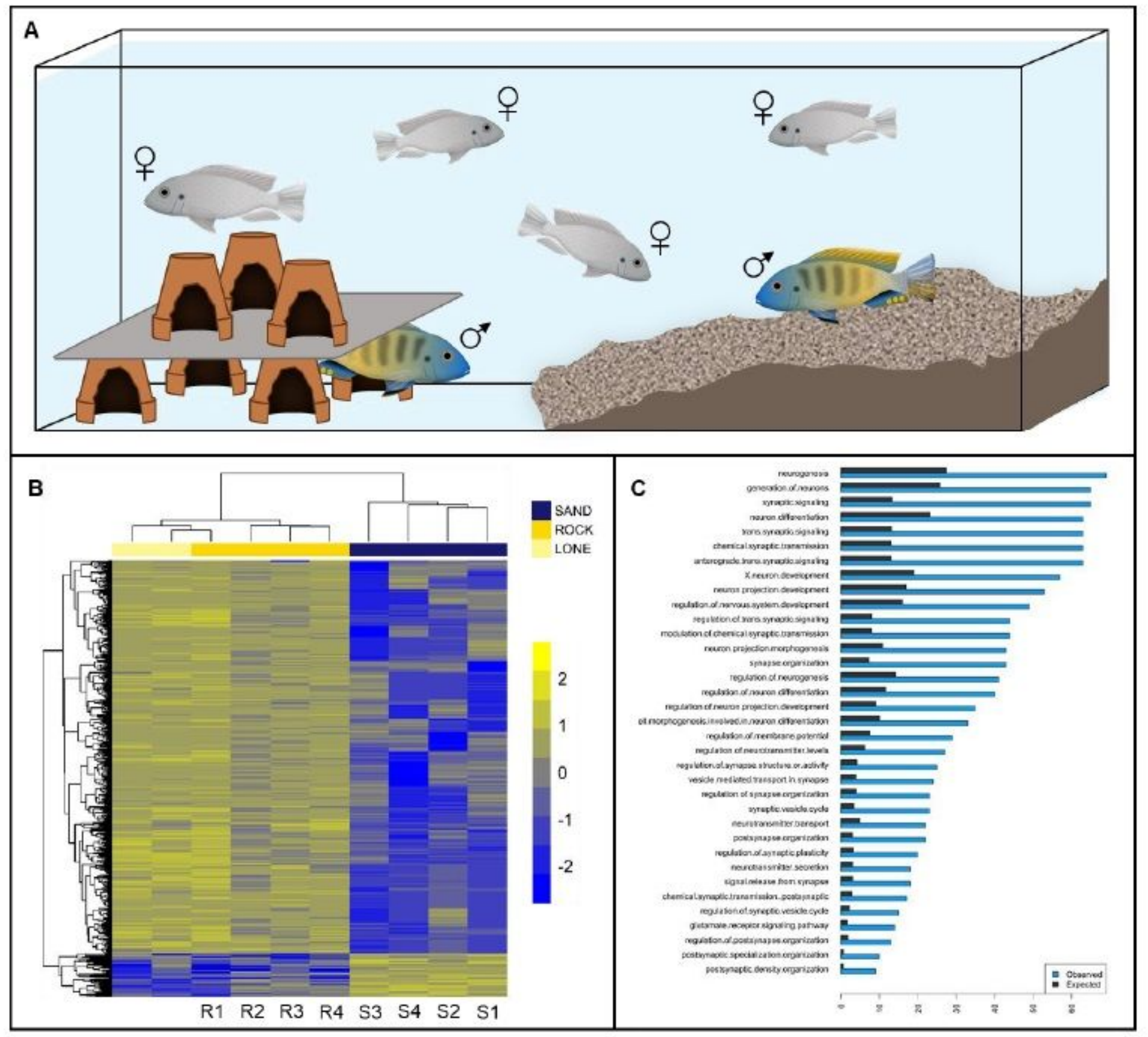

Figure 3

Genomics of divergent social context I (A) Schematic of the social context behavioral paradigm, in which rock-, sand- and rock- $X$ sand- $F 1$ hybrids were evaluated. (B) Heatmap of genes differentially expressed in the brains of F1 males behaving in either rock or sand social contexts. Each row in the heatmap is a gene, each column is an individual. $\mathrm{R}$ individuals are socially rock, $\mathrm{S}$ individuals are socially sand and the numbers indicate sibling pairs. Two F1 males were not paired with other males, and courted females over the rock habitat (lone). All other F1 males $(n=8)$ were introduced to the testing arena in dyads. Notably, male brain gene expression clusters by social context and not fraternal relationships. (C) Gene Ontology (GO) Biological Process terms that show greater than expected overlap between (i) genes differentially 
expressed in the brains of social rock-vs. social sand- males and (ii) genes that are differentiated in the genomes of rock- vs sand- species groups.

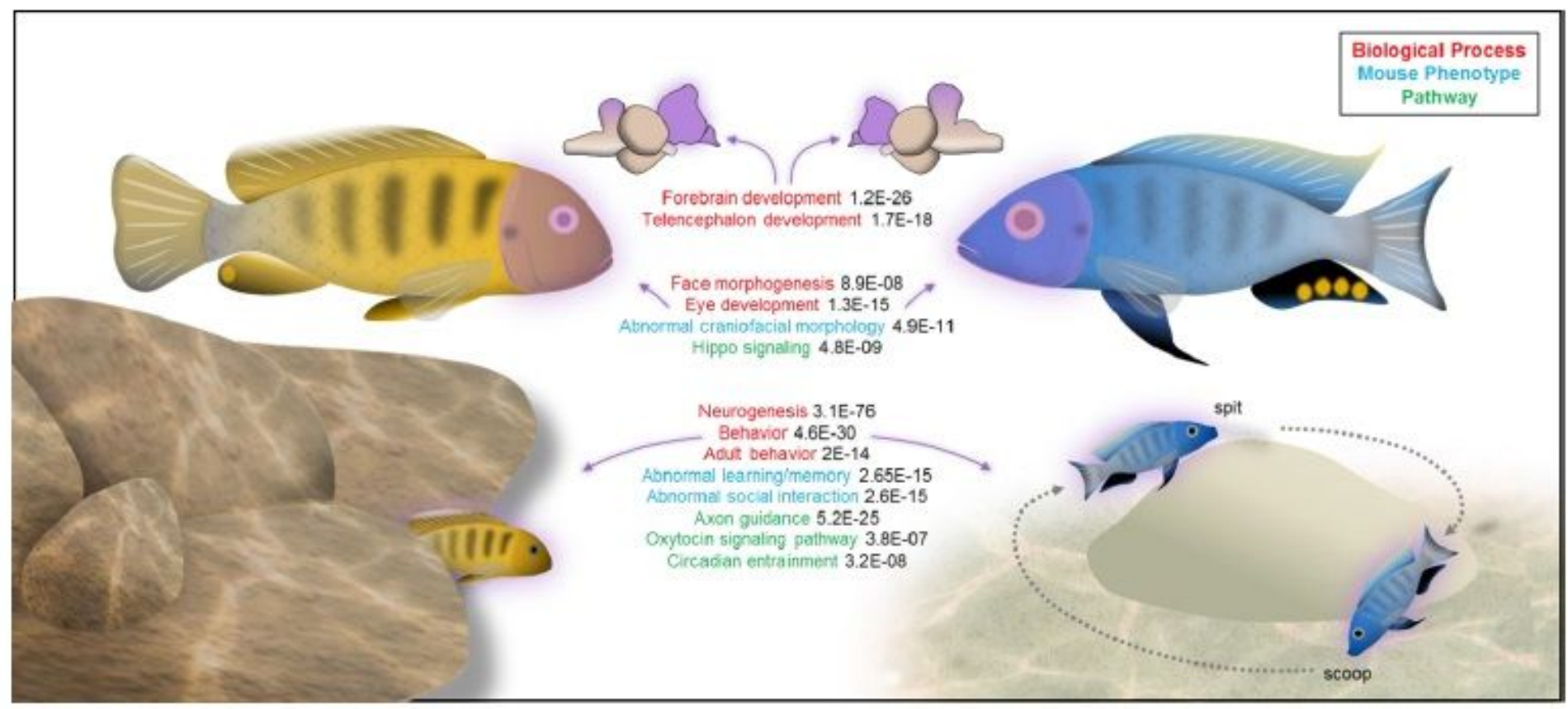

\section{Figure 4}

Genome-enabled discovery of evolutionary change in morphology and behavior. | Summary cartoon synthesizing significant enrichment categories that differentiate the genomes of rock-vs. sand-dwelling Malawi cichlids. Strong and consistent enrichment of craniofacial, neural and behavioral categories motivated follow-on experiments in early brain development (Figure 2) and adult social behavior (Figure $3)$.

\section{Supplementary Files}

This is a list of supplementary files associated with this preprint. Click to download.

- SupplementaryTable1Speciesandseqcharacteristics.xlsx

- SupplementaryTable2genomewideidentityofenrichmentGAtopfunSFARlenhancers.xlsx

- SupplementaryTable3RNAseqDEG.xlsx

- SupplementaryTable4rocksandversuspitcastle.xlsx

- SupplementaryTable5SamplesforRNAseq.xlsx

- supplementaryinformation.pdf 